\title{
An Incremental Method for the Calculation of the Electron Correlation Energy in Metals
}

\author{
Beate Paulus ${ }^{1}$ and Alexander Mitin ${ }^{2}$ \\ Max-Planck-Institut für Physik komplexer Systeme, \\ Nöthnitzer Straße 38, \\ 01187 Dresden, Germany
}

Received ......, 2006; accepted xx, 2006

\begin{abstract}
The method of increments, which is a wavefunction-based correlation method for solids, is extended to metals. Whereas the Hartree-Fock energy is calculated in the infinite periodic solid, the correlation energy of the solid is expanded in terms of localised orbitals or a group of localised orbitals. For the metals the so-called correlation energy increments are determined in finite, properly embedded fragments of the metal. First applications to the ground-state properties of solid mercury yield very good agreement with experiment $[1,2,3]$. In this paper we present the extension of the method to solid barium. Whereas the metallic character in mercury is due to the hybridisation of the $6 s^{2}$ electrons with the $6 p$ orbitals, in barium it is due to the hybridisation with the $5 d$ orbitals. Due to a proper embedding scheme we can to model the electronic structure of bulk barium and In the case of barium we have to use multi-reference methods for the incremental treatment of the correlation energy.
\end{abstract}

Keywords: Ab-initio calculation; Electron correlations; Method of increments; Heavy metals;

PACS: 31.10.+z, 31.25.Nj, 61.50.Ah, 61.50.Lt

\section{Introduction}

It is nowadays possible to calculate properties from first principles for a wide range of materials. This is mainly due to the success of density-functional theory (DFT). But especially for heavier elements the density functional methods have their difficulties due to the approximations in the exchange-correlation functional. In particular for mercury they can not predict the ground-state structure and an reasonable cohesive energy[3].

Here, we want to present an alternative to DFT, invoking the wavefunction-based ab-initio methods of quantum chemistry. To use these methods for solids, it is recommended to transform the extended Bloch states of the solid in localised Wannier states and perform the correlation calculation with the localised orbitals yielding the family of local correlation methods $[4,5,6]$. The method of increments was developed specially for the purpose of calculating the correlation energy of a solid (for a review see [7]). In this approach the correlation energy is calculated in the first step as the sum of independent contributions of translationally invariant localised orbitals or groups of localised orbitals. As corrections the non-additive parts of two, three or more localised orbitals are

\footnotetext{
${ }^{1}$ Corresponding author. E-mail: beate@pks.mpg.de

${ }^{2}$ present address: Department of Computer Science, 7 Universitetsparken 1 7, DK-2100 Copenhagen
} 
added. If the group of localised orbitals is chosen reasonably this partitioning of the correlation energy of the solid yields a quickly converging series. Due to the partitioning it is necessary that the correlation method used for the individual increments be size-extensive, but no further restrictions are necessary.

The method of increments has been successfully applied to ground-state properties of insulators and semiconductors ranging from strongly bound ionic (including transition-metal oxides and lanthanide nitrides and oxides) or covalent systems to weakly bound van der Waals solids. The generalisation to metals has been first applied to solid mercury[1, 2, 3], where a single-reference coupled cluster approach was used for the correlation calculation. Here we present preliminary results for solid barium, where in contrast to mercury the metallic character is $d$-like and a multireference correlation treatment is necessary.

The paper is organised as follows: In the next section the method of increments for metals is presented and the embedding for the metals is described. In Section III the Hartree-Fock results for mercury and barium are discussed. In Sec. IV the free barium dimer is compared to the embedded two-body increment of solid barium. Conclusion and outlook follows in Sec. V.

\section{The method of increments for metals}

We are setting up a many-body expansion for the correlation energy of the solid per unit cell (u.c.) of the form

$$
E_{\mathrm{corr}}^{\mathrm{solid}}=\sum_{I \in \text { u.c. }} \epsilon_{I}+\frac{1}{2} \sum_{\substack{I \neq J \\ I \in \text { u.c. } \\ J \in \text { solid }}} \Delta \epsilon_{I J}+\frac{1}{6} \sum_{\substack{I \neq J \neq K \\ I \in \text { u. c.id } \\ J, K \in \text { solid }}} \Delta \epsilon_{I J K}+\cdots .
$$

The partitioning is done in this way, that the exact correlation energy of the solid is given by a sum of all increments. Whereas the first index $I$ runs only over the centres of the unit cell, all other indices $J, K$ run over the whole solid. The two-body increment $\Delta \epsilon_{I J}$ describes the non-additive part of the excitations involving centre $I$ and $J$ :

$$
\Delta \epsilon_{I J}:=\epsilon_{I J}-\left(\epsilon_{I}+\epsilon_{J}\right) .
$$

Higher order increments are defined analogously. For the three-body energy increment we get

$$
\Delta \epsilon_{I J K}:=\epsilon_{I J K}-\left(\epsilon_{I}+\epsilon_{J}+\epsilon_{K}\right)-\left(\Delta \epsilon_{I J}+\Delta \epsilon_{J K}+\Delta \epsilon_{I K}\right) .
$$

All corresponding two-body and one-body increments are subtracted, only the pure 3-body effects are included in $\Delta \epsilon_{I J K}$.

This partitioning of the correlation energy is only sensible if the series converges quickly enough, both with the order of increments (i.e., 3-body increments should be significantly smaller than two-body increments, etc.) and with the distance of the orbital groups involved in the increments. Increments involving distant orbital groups must decay faster than the number of increments increases in a 3-dimensional system. For a semiconductor or an insulator, increments with distant orbital groups interact only via van der Waals and thus decay like $r^{-6}$, whereas the number of pairs grows with $r^{2}$, so an overall decay of $r^{-4}$ guarantees the convergence with respect to the distance of orbital groups.

A direct transfer of this approach to metallic systems is not possible, however, since localised orbitals become very long-range entities here and a many-body expansion in terms of such orbitals cannot be expected to have useful convergence characteristics. In order to make the expansion still computationally feasible, we have suggested[1,2] to start from suitable model systems where long-range orbital tails are absent, and to allow for delocalisation only successively in the course of the expansion; more specifically, when calculating pair contributions for a given orbital group 
combination $(I, J)$, we allow for delocalisation $I \rightarrow J$ and $J \rightarrow I$, and similarly with the 3body terms we allow for delocalisation over the triples of atoms, etc. It is clear that the final result is not affected, only the convergence properties of the many-body expansion are changed. As an additional advantage, we can calculate individual terms of the expansion from (suitably modelled/embedded) finite clusters of reasonable size. In the case of mercury and barium, for example, we can force localisation of the solid by using a $s$-type atomic basis set for describing the valence-electron system. This way, delocalisation due to $s p$-mixing or $s d$-mixing is avoided, but still each atom has its correct crystal surroundings concerning the electrostatic interaction. Due to the metallic embedding scheme even the conduction bands are localised in the central region. The incremental expansion has to take care subsequently of the non-additive effects of correlation and delocalisation.

\section{Hartree-Fock results for mercury and barium}
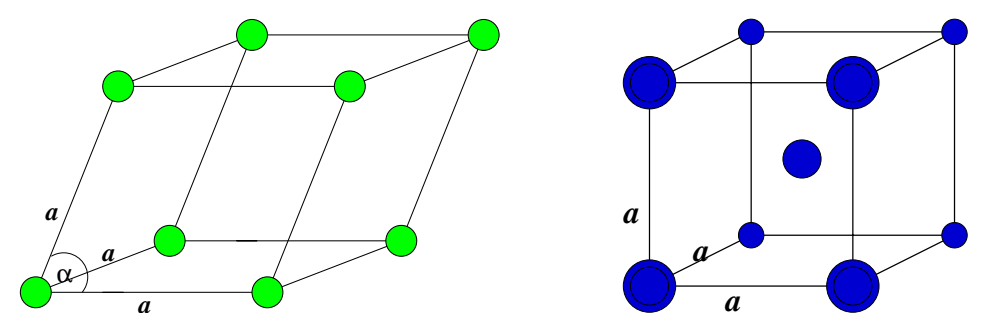

Figure 1: The rhombohedral lattice structure (left) of $\mathrm{Hg}$ and the bcc one (right) for $\mathrm{Ba}$

The method of increments works well only for the expansion of the correlation energy, because the correlation hole in a solid is of short-range. The Hartree-Fock part has to be calculated in the extended systems to describe the long-range Coulomb interaction properly. Therefore we perform periodic Hartree-Fock calculations using the program package CRYSTAL98 [8].

Mercury condenses below $233 \mathrm{~K}$ into the rhombohedral structure, which can be described by two parameters, a bond length $a=3.005 \AA$ and rhombohedral angle $\alpha=70.53^{\circ}$ [9] (see Fig.1). Barium has a body-centred cubic (bcc) structure with a lattice constant of $5.0227 \AA$ [9] which corresponds to a nearest neighbour distance of $4.3498 \AA$ (see Fig.1).

The chemically inactive $[\mathrm{Kr}] 4 d^{10} 4 f^{14}$ core of $\mathrm{Hg}$ and $\mathrm{Ba}$ is simulated by an energy-consistent scalar-relativistic pseudopotential (PP)[10]. The basis set used is a contracted Gaussian type orbital (CGTO) set, $(6 s 6 p 6 d) /[5 s 4 p 3 d]$ for $\mathrm{Hg}[2]$ and $(7 s 8 p 3 d) /[4 s 4 p 3 d]$ for Ba (see Table 1), both optimised for the experimental crystal structure. The Hartree-Fock band structure of mercury and barium is plotted in Fig.2. For mercury the filled $5 d$ bands show nearly no dispersion and lie about 0.5 Hartree below the Fermi level, the metallic character arises from the $6 s p$ mixing at the L-point. At the $\Gamma$-point a gap of more than 1 Hartree occurs. The valence band has predominantly $s$-character. This is also true for barium. But in contrast to mercury the metallic character arises due to $5 d-6 s$ mixing especially at the H-point. The unoccupied $5 d$ shell shows a dispersion of about 0.5 Hartree and the centre of the $5 d$ bands lies only about 0.2 Hartree over the Fermi level. The $6 p$ band is centred above the $5 d$ bands. The main difference between the two band structure is the position of the $5 d$ bands. This is also seen in the electronic density of states (see Fig.3). Whereas in mercury the density of state is half $s$ and half $p$-like at the Fermi level, for barium we see two third $d$ character and one third $s p$ character. 


\begin{tabular}{||r|r||r|r||r|r||}
\hline$s$-exp. & contr. & $p$-exp. & contr. & $d$-exp. & contr. \\
\hline 13.329420 & 0.078383 & 8.497514 & 0.276899 & 2.603516 & 1.000000 \\
11.319460 & -0.138024 & 7.646964 & -0.572659 & 1.055567 & 1.000000 \\
3.579639 & 1.131201 & 5.985080 & 0.429937 & 0.110000 & 1.000000 \\
2.637996 & -1.492940 & 2.422947 & -0.354379 & & \\
0.918054 & 1.000000 & 0.883951 & -0.198726 & & \\
0.340000 & 1.000000 & 0.651175 & 1.000000 & & \\
0.050000 & 1.000000 & 0.260000 & 1.000000 & & \\
& & 0.060000 & 1.000000 & & \\
\hline
\end{tabular}

Table 1: The crystal-optimised basis set for Ba for the 10-valence-electron energy-consistent scalarrelativistic pseudopotential $(\mathrm{PP})$.
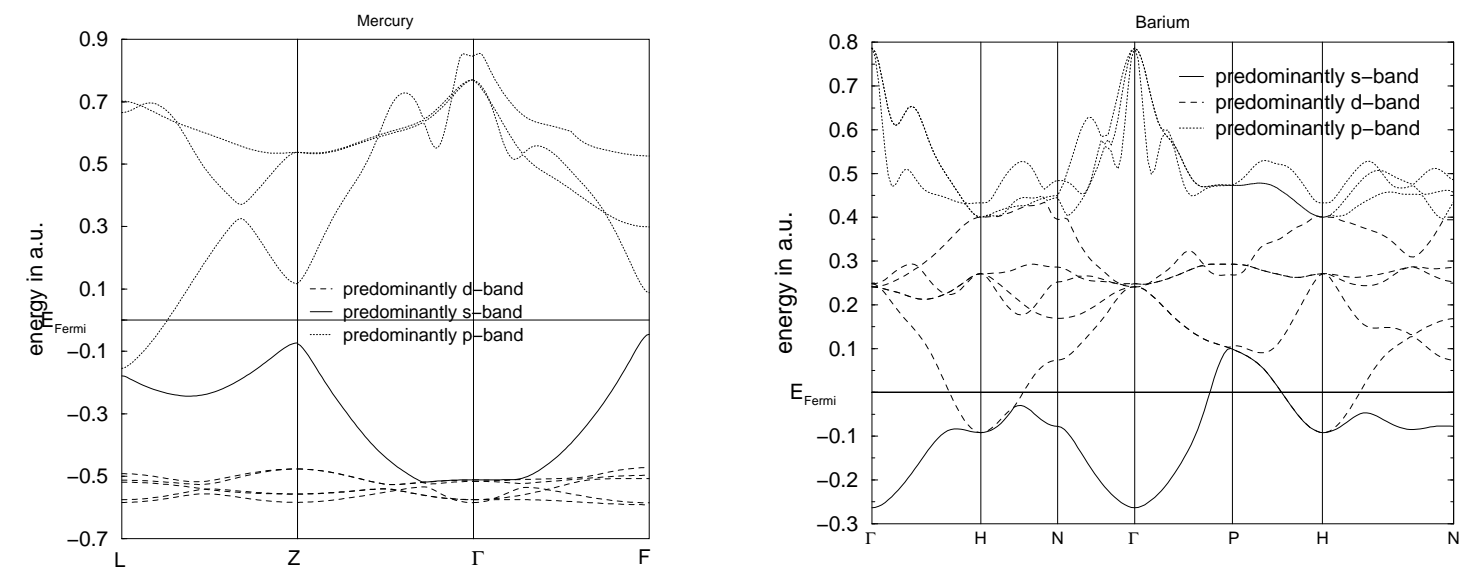

Figure 2: The Hartree-Fock band structure of $\mathrm{Hg}$ in the rhombohedral structure (left) and of $\mathrm{Ba}$ in the bcc structure (right).
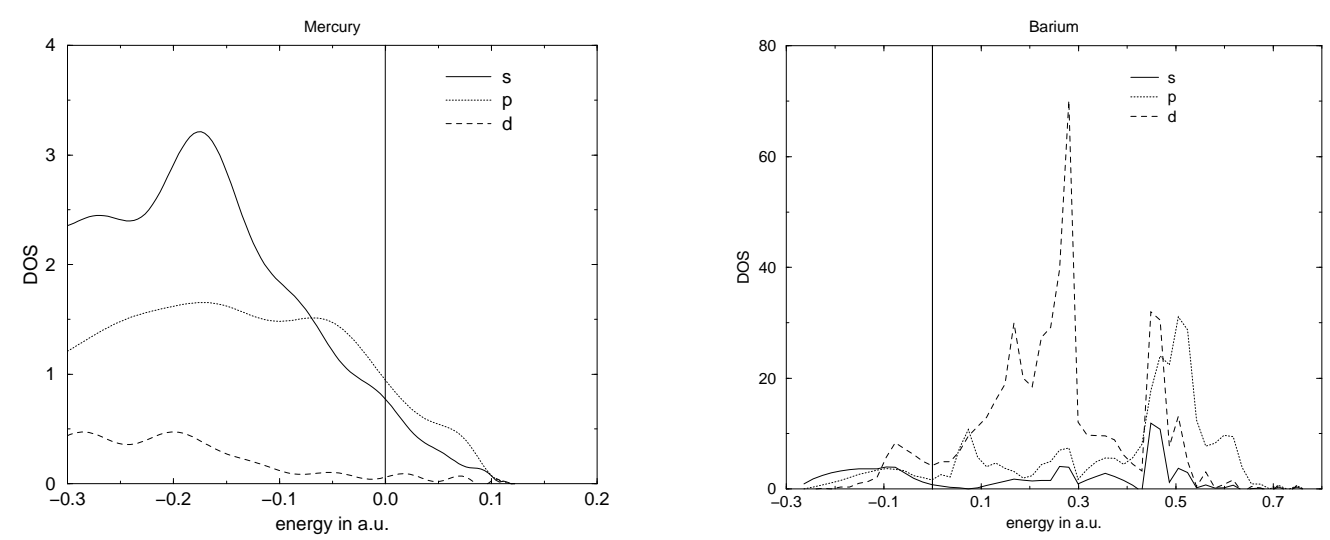

Figure 3: The Hartree-Fock density of states of $\mathrm{Hg}$ in the rhombohedral structure (left) and of Ba in the bcc structure (right). The Fermi energy is in both cases at zero. 


\begin{tabular}{|c|c|c|}
\hline method & $R_{\mathrm{e}}[\AA]$ & $\mathrm{D}_{\mathrm{e}}[\mathrm{eV}]$ \\
\hline $\operatorname{CCSD}(\mathrm{T})$ & 5.162 & 0.110 \\
\hline CASPT2 & 5.210 & 0.120 \\
\hline CIPSI[12] & 4.881 & 0.202 \\
\hline LDA[13] & 4.749 & 0.355 \\
\hline $\operatorname{CCSD}(\mathrm{T})[13]$ & 5.079 & 0.1368 \\
\hline
\end{tabular}

Table 2: The equilibrium distance and dissociation energy for the barium dimer with different methods in comparison to the values from the literature.

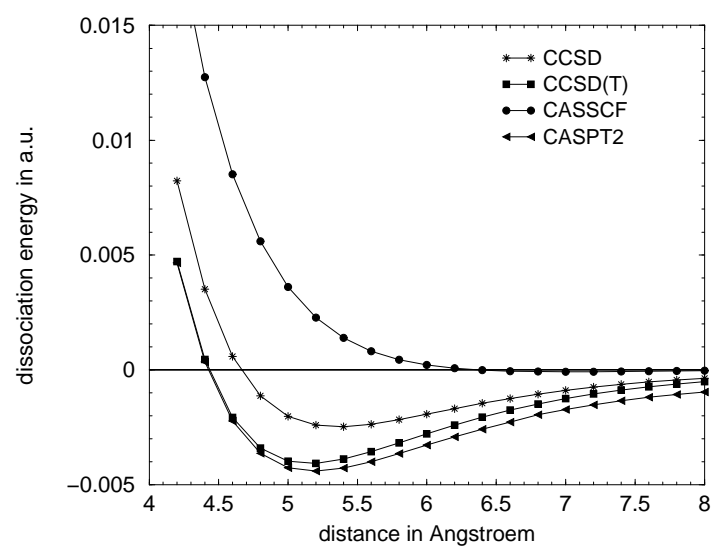

Figure 4: The dissociation curve of Barium dimer for the single-reference coupled cluster method and the complete active space perturbation theory second order

\section{Comparison between free and embedded $\mathrm{Ba}_{2}$}

Whereas for the free mercury dimer it is known, that only highly correlated correlation methods yield a reasonable result for the equilibrium distance and binding energy, less data are available in the literature for the free barium dimer. Therefore we determined in a first step the equilibrium distance and dissociation energy for the barium dimer with different methods (see Fig. 4). We applied the scalar-relativistic 10-valence-electron pseudopotential[10] with the corresponding uncontracted (9s9p6d4f) basis set optimised for MP2 calculations[10]. Additionally two $g$ functions with the exponents 0.4 and 0.8 are added. All 10 valence electrons per atom are correlated. The active space in the CAS calculations is 2 active orbitals per atom. The dissociation curves are BSSE corrected. The program package Molpro[11] was used for all correlation calculations. In Table 2 we summarised our results and compared with the values in the literature.

We only performed the calculations for the free dimer to test our methods and to analyse the electronic structure at the equilibrium distance and at the nearest neighbour distance of the solid. Our CCSD(T) results agree well with the published one[13]. Due to the somewhat smaller basis set our equilibrium distance is a little bit larger and the dissociation energy smaller. The free barium dimer can be well described with a single reference method, a multi-reference treatment like the CASPT2 here yielding quite similar results to the $\operatorname{CCSD}(\mathrm{T})$ treatment. If we analyse the CASSCF wavefunction for an active space of 2 orbitals per barium atom, we see (Table 3 ) for the equilibrium distance, that the leading configuration (coefficient 0.95) is a bonding $\sigma$ orbital, followed by configurations where 2 electrons are excited to a $\pi$-like orbital (coefficient 0.12). For 


\begin{tabular}{||ll||l|l|l|l||}
\hline $\mathrm{Ba}_{2}$ & & free & free & emb. & emb. \\
distance & & $5.00 \AA$ & $4.35 \AA$ & $4.35 \AA$ & $5.028 \AA$ \\
\hline CI-coeff. & & $0.95(\sigma)$ & $0.95(\sigma)$ & $0.78(\sigma)$ & $0.86(\sigma \delta)$ \\
& & $0.12(\pi)$ & $0.20(\pi)$ & $0.49(\delta)$ & $0.37(\sigma \pi)$ \\
& & $0.12(\pi)$ & $0.20(\pi)$ & $0.20(\delta)$ & $0.26(\sigma \pi \delta)$ \\
\hline val. pop. & $\mathrm{s}$ & 1.84 & 1.82 & 0.52 & 0.88 \\
& $\mathrm{p}$ & 0.13 & 0.13 & 0.11 & -0.02 \\
& $\mathrm{~d}$ & 0.03 & 0.05 & 1.27 & 1.04 \\
\hline
\end{tabular}

Table 3: The three leading CI coefficient of an CASSCF calculation of the Ba dimer at different distances and in comparison of the free dimer and the in a fragment of a the bcc solid embedded dimer. The predominant character of the configuration is given in parenthesis. The valence populations per barium atom determined with a Mulliken population analysis for the CAS wavefunction splitted according to the angular momentum.

the equilibrium distance also configurations with single occupied orbitals are important (coefficient $\approx 0.12$ ).

If we regard the free dimer at a distance of $4.35 \AA$, which is the nearest neighbour distance in the solid, not much is changed for the CAS-wavefunction. Still the leading configuration (coefficient 0.95 ) is a $\sigma$ bond, whereas the importance of configurations with $\pi$-like orbitals increases (coefficient 0.20 ), but the coefficients of configurations with single occupied orbitals are below 0.05. Having in mind the strong $d$ character of the conduction band in solid barium, it can not be generated just by reducing the distance between the atoms.

Therefore we generate an embedding for the barium dimer to simulate the solid structure. Around the dimer with the nearest neighbour distance $(4.35 \AA)$ we arrange 20 barium atoms in the solid bcc structure and around the second nearest neighbours (5.028 $\AA$ ) we arrange 22 barium atoms. The embedding atoms are described with a large-core pseudopotential[10], only the 2 valence electrons are treated explicitly. To prevent delocalisation to the embedding region, we supply only a minimal $s$ basis set to the embedding atoms. The procedure is as follows. First all atoms are described with minimal basis set only and the orbitals are localised according to the Foster-Boys procedure. The orbitals of the embedding are kept frozen whereas the orbitals on the two central atoms are reoptimized with the full basis set $(9 \mathrm{~s} 9 \mathrm{p} 6 \mathrm{~d} 4 \mathrm{f})$. The character of the orbitals changes from purely $s$-like to a significant admixture of $d$ and $p$ character. Performing a CASSCF calculation with an active space of only 2 orbitals per barium atom, the leading coefficient the $\sigma$-bond is now only 0.78 , and the next important configurations are not longer $p$-like as in the free dimer, but $d$-like with an coefficient of 0.49. Due to the embedding we can now model the electronic structure of the solid. In the embedded dimers the character of the wave-function is a mixture of $s$ and $d$ character of about the same ratio as the mixture in the density of states at the Fermi level in the periodic solid.

\section{Conclusion and Outlook}

We have here presented the extension of the method of increments to metals. Whereas for solid mercury a single-reference correlation method is sufficient, for barium it is necessary to use a multireference scheme due to the quasi-degenerate empty $5 d$ shell. That is qualitatively different to the barium dimer, where the $5 d$ orbitals have no significant role in the binding which therefore can be well described with a single-reference method. The lowering of the $d$ level is not caused by the 
shortening of the distance between the barium atoms, but due to three-dimensional packing in bcc solid. We have shown, that we can model the situation in the solid via a embedding describing the electrostatic interactions but not allowing for a delocalisation in the embedding region.

With these results we can now start to perform an incremental expansion for the correlation energy of solid barium. Without changing the general formalism it is possible to apply the method of increments to quasi-degenerate systems. The correlation energy is now partitioned into a CASSCF part, which describes the static correlations and yields the correct dissociation limit, and a MR part for the dynamical correlations. In the case of metals, we select an active space for the CASSCF calculation including the important bands around the Fermi level. The dynamical correlations are treated on top of the MCSCF wave-function with an approximately size-extensive multi-reference correlation method. For a one-dimensional model system of Li-atoms we used an MR averaged coupled pair functional method and achieved a converging incremental expansion[14]. Work is underway in our group to extend the multi-reference method of increments to solid barium by using a multi-reference perturbation theory for the dynamical correlations.

\section{References}

[1] B. Paulus and K. Rosciszewski, Chem. Phys. Lett., 394, 96-100 (2004).

[2] B. Paulus, K. Rosciszewski, N. Gaston, P. Schwerdtfeger and H. Stoll, Physical Review B,70, 165106 (2004).

[3] N. Gaston, B. Paulus, K. Rosciszewski, P. Schwerdtfeger and H. Stoll, submitted to Physical Review B (2006).

[4] G. Stollhoff and P. Fulde, J. Chem. Phys., 73 (1980) 4548.

[5] P. Pulay, Chem. Phys. Lett., 100 (1983) 151.

[6] H. Stoll, Phys. Rev. B, 46 (1992) 6700.

[7] B. Paulus, Physics Reports, 428, 1 (2006).

[8] V.R. Saunders, R. Dovesi, C. Roetti, et. al. program Crystal 98, Theoretical Chemistry Group, University of Torino (1998).

[9] CRC Handbook of Chemistry and Physics, CRC Press, New York (1997).

[10] The Stuttgart Pseudopotential Library, H. Stoll, M. Dolg and others, (see http://www.theochem.uni-stuttgart.de/pseudopotentials).

[11] MOLPRO version 2002.6 - a package of ab-initio programs written by H.-J. Werner and P. J. Knowles and R. Lindh and M. Schütz and others, (see http://www.molpro.net), Birmingham, UK, 2003.

[12] A.R. Allouche, M. Aubert-Frécon, G. Nicolas and F. Spiegelmann, Chem. Phys. 200, 63 (1995).

[13] N. Gaston and P. Schwerdtfeger, Phys. Rev. B, in press (2006).

[14] B. Paulus, Chem. Phys. Lett., 371 (2003) 7. 